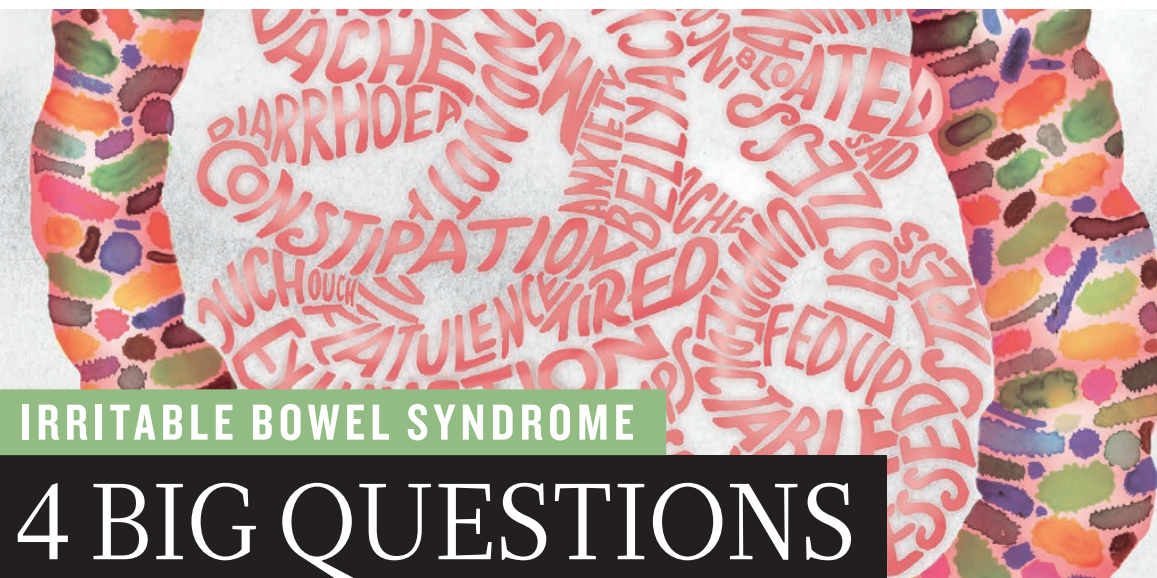

There is a long list

of unknowns about

the causes and

symptomatology of

irritable bowel syndrome

(IBS). But the research

challenges can be distilled

into four key areas.

BY MICHELLE GRAYSON

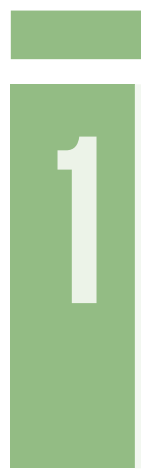

QUESTION

What are the

underlying

mechanisms of the

syndrome?

What is the

relationship between

the disorder and

mental health?

\section{WHY IT MATTERS}

WHAT WE KNOW

The variety of symptoms and of patient responses to treatment suggest that there are multiple underlying causes of IBS. Identifying specific mechanisms will help researchers to develop better treatments.
The gut can only react to insults in certain ways (for example, diarrhoea, constipation or cramps). But these symptoms could be the end point of several different diseases.

\section{NEXT STEPS}

For treatment purposes, it is important to know whether IBS starts in the gut and then causes psychological issues, or whether IBS symptoms have a neurological trigger.
Anxiety and depression sometimes accompany IBS. The serotonin system is implicated in the syndrome, and plays a part in neurological conditions. IBS is more common in service personnel who have posttraumatic stress disorder.
Researchers have already identified distinct causes of IBS, such as bile-acid malabsorption. Large-scale initiatives such as Europe's GENIEUR consortium are trying to tease out genetic and epigenetic contributions.

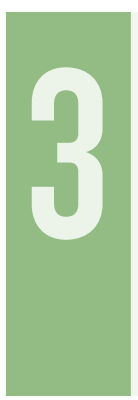

How important is diet in driving IBS?

Can the syndrome be definitively diagnosed?
Diet seems an obvious starting point for treating IBS. Care will be improved with a better understanding of which subset of patients could benefit from dietary interventions and why.

An IBS diagnosis is based on symptoms and on the elimination of other disorders. This is stressful for patients, frustrating for clinicians and inefficient for the health-care system.
The fermentation of certain food components by microorganisms in the gut exacerbates symptoms. Diet can ease these, but there is little evidence of a causal link.
Better grouping of patients by phenotype will help researchers to pick out signals in large-scale trials. In the meantime, clinicians need to be careful not to assume that patients have mainly a psychological problem.
Larger, more rigorous trials will help to isolate the effect of food components. By working closer together, microbiologists, gastroenterologists and nutritionists could improve knowledge of the behaviour of the gut microbiota.
Attempts to develop biomarkers for IBS have been disappointing. Breath tests and diagnostics that involve serum biomarkers are in development, but these have yet to translate to the clinic and many researchers remain sceptical.
Clearer patient phenotypes will help researchers to identify more specific biomarkers, which can also be used to stratify patients for clinical testing of targeted drugs. 